\title{
Bilateral Telemanipulation: Improving the Complementarity of the Frequency- and Time-Domain Passivity Approaches
}

\author{
Michel Franken, Bert Willaert, Sarthak Misra, and Stefano Stramigioli
}

\begin{abstract}
Passivity of bilateral telemanipulation systems ensures stability of the interaction with such systems. In the frequency domain, passivity of a linear time invariant approximation of the system can be designed for a considered set of operating conditions. Non-linear control structures have been proposed that enforce passivity of the system in the time domain. In this paper, extensions are proposed that increase the complimentarity of the frequency- and timedomain approaches. The combination of both approaches allows a guaranteed measure of transparency to be designed in the frequency domain for a desired set of operating conditions. For operating conditions outside the desired set, stable interaction is guaranteed by the non-linear passivity enforcing control structure. Simulation results of the combined approach are presented that show that the stability properties of the bilateral controller designed in the frequency domain are improved and the transparency properties are improved with respect to those of the standard passivity-enforcing algorithm in the timedomain.
\end{abstract}

\section{INTRODUCTION}

Bilateral telemanipulation systems reflect force information about the remote interaction between the slave system and the environment to the user. This bidirectional coupling between the user and the environment comprises a closed chain with multiple possible unknown and time-varying components, e.g. the user and environment impedance. A major research topic is therefore how to optimize transparency, [1], while guaranteeing stability of the coupled system under all operating conditions. With respect to the stability issue various passivity-based approaches have been introduced in literature. A passive system cannot generate energy and the interconnection of a passive system with any other passive system is guaranteed to be stable [2]. As the environment can be assumed to be passive and humans can interact very well with passive systems [3], a passivity-based approach is an elegant solution to the stability problem.

We identify two major categories in the control of bilateral telemanipulation systems which are centered around the concept of passivity of the system:

1) Approaches that design bilateral controllers in the frequency domain (FD), based on Linear Time Invariant (LTI) models of the system, e.g. [1], [4]-[6]

2) Approaches that implement non-linear control structures that enforce passivity of the bilateral controller in the time domain (TD), e.g. [7]-[10]

M. Franken, S. Misra, and S. Stramigioli are affiliated with the MIRA Institute for Biomedical Technology and Technical Medicine, University of Twente, The Netherlands.

B. Willaert is affiliated with the Department of Mechanical Engineering, K.U. Leuven, Belgium.

E-mail:m.c.j.franken@utwente.nl,bert.willaert@mech.kuleuven.be
Naturally, each approach has its benefits and drawbacks, making it more or less suited to deal with specific problems. For instance, the FD approaches result in linear controllers of which the stability and transparency properties are easier to compute. Whereas the TD approaches can better deal with all kinds of non-linear and time-varying effects, e.g. nonlinear device dynamics, time-varying communication delays including package loss, and can accommodate a wide range of bilateral controllers [8], of which the passivity-properties can not necessarily be analyzed in the FD.

In this paper we want to show that both approaches can be complimentary to a large extent when considering LTI systems. In the FD design phase normally all possible operating conditions need to be taken into account, which results in restrictions on the control parameters of the system and thus limits the achievable transparency. The combination with a passivity enforcing control structure in the TD alleviates this restriction as it guarantees stability and allows a restricted set of operating conditions to be taken into account in the FD design phase. In reverse the combination with a FD design phase allows to better quantify the transparency properties of the TD algorithm as it will provide insight in the operating conditions that activate the TD algorithm.

The paper is organized as follows: The model of the system which we will use for the analysis and numerical simulations is introduced in Section II. The concept of passivity will be discussed in Section III and also recent work on FD and TD passivity-based approaches will be discussed. Section IV describes the desired complimentary effect of the two passivity-based approaches of Section III. Section V introduces the extensions to the TD passivity-based approach needed to establish that complimentary effect. An example in which the FD and TD approaches are combined will be discussed in Section VI. In Section VII, trade-offs between transparency and stability that persist are indicated. The paper concludes in Section VIII.

\section{SYSTEM DESCRIPTION}

In this section, we will present the description of the telemanipulation chain that we will use throughout the paper. For our analysis we will assume that both the master and the slave system are one degree of freedom rigid bodies with mass $M$ and subjected to viscous friction $B$ :

$$
\begin{aligned}
F_{h}(t)+F_{m}(t) & =M_{m} \ddot{q}_{m}(t)+B_{m} \dot{q}_{m}(t) \\
F_{e}(t)+F_{s}(t) & =M_{s} \ddot{q}_{s}(t)+B_{s} \dot{q}_{s}(t)
\end{aligned}
$$

where $F$ and $q$ represent forces and positions, respectively. The subscripts $h, m, s$, and $e$ indicate the human operator, 
the master device, the slave device, and the environment, respectively.

The implemented bilateral controller is a Position-Force (P-F) controller. A perfect communication network is assumed (no time delay and losses). This choice is made as the FD passivity analysis of this controller can still be relatively easy performed analytically. However, the results presented in this paper can be established for any LTI bilateral controller. The P-F controller is:

$$
\begin{aligned}
F_{m}(t) & =\lambda F_{e}(t) \\
F_{s}(t) & =K_{p}\left(\mu q_{m}(t)-q_{s}(t)\right)-K_{v} \dot{q}_{s}(t)
\end{aligned}
$$

where $K_{p}, K_{v}$ are the parameters of the position controller at the slave side and $\mu, \lambda$ the scaling factors applied to the position and force commands.

\section{PASSIVITY}

The underlying condition for passivity is that the energy that can be extracted from the system at any time is bounded by the energy that was injected into the system and the energy that was initially stored in the system [2]:

$$
\int_{0}^{t}-F(t)^{T} \dot{q}(t) d t \geq-E(0),
$$

where $F(t)$ and $\dot{q}(t)$ are the forces and velocities at the interaction points, respectively. $E(0)$ is the energy initially stored in the system and assumed to be zero. The telemanipulation system is a two-port system so (3) can be written as:

$$
\int_{0}^{t} F_{h}(t)^{T} \dot{q}_{m}(t)+F_{e}(t)^{T} \dot{q}_{s}(t) d t \geq 0 .
$$

where the sign change is due to the use of $F_{h}(t)$ and $F_{e}(t)$ according to (1). If a system is non-passive it is said to generate "virtual" energy and this energy can potentially destabilize the system.

\section{A. Frequency Domain Passivity-Based Design}

Passivity of a two-port LTI-system can be checked in the FD using Raisbeck's passivity criterion [11]. However, for many LTI bilateral controllers the range of allowed parameter settings based on this criterion is extremely limited if at all existing. For the system of Section II Willaert et al. [6] proof that it can never comply with Raisbeck's passivity criterion. Consequently, two-port passivity of telemanipulation systems is not very useful as a design tool in the FD due to too restrictive sufficient conditions given by the used criterion.

However, the guaranteed stability due to passivity remains an attractive property for bilateral systems. This inspired researchers, e.g. [4]-[6], to incorporate more information about the application of the system in the design phase and apply passivity-based designs on these extended models.

The operating conditions of a telemanipulation system specify everything that is related to the interaction between the telemanipulation system and the user/environment, e.g. control parameters, device impedances, time-variant impedances of the user/environment. A set of operating conditions, e.g. the range of impedances of the environment, can be composed of all operating conditions that can/will occur. The FD approaches discussed below analyze the passivity of part of the telemanipulation system for a considered set of operating conditions. Transparency of the system is optimized given the boundary condition of passivity.

The Absolute Stability approach, based on the work of Llewellyn [12], derives control parameters that result in a passive one-port system when the telemanipulation system is terminated by any passive impedance at either side. Such a system is guaranteed to be stable as long as there is no direct interaction between the user and the environment. Willaert et al. [6] calculated that the system of Section II is absolute stable if the hardware and control parameters comply with the following two conditions:

$$
\begin{aligned}
\mu \lambda & \leq \frac{4 B_{m}}{B_{s}+K_{v}} \\
\mu \lambda & \leq \frac{B_{m}\left(B_{s}+K_{v}\right)}{M_{s} K_{p}}
\end{aligned}
$$

The Absolute Stability approach alleviates the restrictions on the control parameters with respect to the two-port LTI passivity condition. This is possible as it assumes the absence of a secondary interaction path between the user and the environment and is an example of a restricted set of operating conditions for the design phase. However, the Absolute Stability approach still considers an infinite range for the impedance of the human operator as well as for the environment. In real applications, the range of impedances that can/will be encountered will always be restricted/bounded in some way. Recent work has focussed on including such bounds in the design phase, e.g. [4], [6], [13]

The Bounded Environment Passivity approach [6] can be used to compute control parameters for which the one-port system composed of the telemanipulation system and environment is passive. In the analysis a class of impedances is considered and bounds can be incorporated on the magnitude of each element. For the system of Section II, the following bounds [6] can be derived for environments characterizable as a pure spring model by considering the maximum stiffness, $K_{e}^{\max }$, of the environment:

$$
\begin{aligned}
& B^{l i m}=\min \left[\sqrt{2\left(K_{p}+K_{e}^{\max }\right) M_{s}}, 2 \sqrt{K_{p} M_{s}}\right] \\
& 0 \leq\left(B_{s}+K_{v}\right) \leq B^{l i m} \Rightarrow \mu \lambda \leq \frac{B_{m}\left(B_{s}+K_{v}\right)}{M_{s} K_{p}}\left[1+\frac{K_{p}}{K_{e}^{m a x}}-\frac{\left(B_{s}+K_{v}\right)^{2}}{4 K_{e}^{m a x} M_{s}}\right] \\
& \left(B_{s}+K_{v}\right) \geq B^{l i m} \text { and } K_{e}^{\max } \leq K_{p} \quad \Rightarrow \quad \mu \lambda \leq \frac{B_{m}\left(K_{p}+K_{e}^{\max }\right)^{2}}{\left(B_{s}+K_{v}\right) K_{p} K_{e}^{\max }} \\
& \left(B_{s}+K_{v}\right) \geq B^{l i m} \text { and } K_{e}^{\max }>K_{p} \quad \Rightarrow \quad \mu \lambda \leq \frac{4 B_{m}}{\left(B_{s}+K_{v}\right)}
\end{aligned}
$$

where the ratio between the viscous friction $B_{s}+K_{v}$ in the slave system and $B^{l i m}$ and the ratio between $K_{p}$ and $K_{e}^{\max }$ are used to select a boundary condition for $\mu \lambda$. As detailed by Willaert et al. [6], comparing (6) with (5) shows that assuming a maximum stiffness of the environment can have a relaxing effect on the allowed parameter settings of the bilateral controller.

However, a system designed using these approaches is only passive when all physical environments that can be encountered during operation comply with the assumed classes and/or bounds. When other environments can be 
encountered, the system is not guaranteed to be passive, e.g. when interacting with stiffnesses higher than the assumed $K_{e}^{\max }$, and is thus potentially unstable.

\section{B. Time Domain Passivity Control}

A different approach to ensure passivity of a telemanipulation system would be to apply a non-linear control algorithm that prevents non-passive behavior of the bilateral controller. The class of possible solutions we focus on here are based on Time Domain Passivity Control (TDPC) by damping injection. This was initially proposed by Ryu et al. [10] and extended/different implementations have been proposed to extend the application of this approach by e.g. Artigas et al. [7] and Franken et al. [8].

The basic concept of this class of TDPC is to monitor an appropriate energy balance for the system controlled by a bilateral controller. Non-passive behavior of the system is detected by the monitored balance becoming negative and a modulated damper at the master side is activated to modify the commands of the bilateral controller to maintain a neutral energy balance.

This class of TDPC algorithms is highly applicable to impedance type displays (velocity input, force output causality). For this class of systems, the energy exchange between the bilateral control algorithm and the master and slave devices can be exactly computed as [14]:

$$
\Delta H_{I}(k)=-F_{a}(\bar{k}) \Delta q_{a}(k)
$$

where $\Delta H_{I}(k)$ is the energy exchanged between the control algorithm and the physical device during sample period $\bar{k}$, $F_{a}(\bar{k})$ is the force applied by the actuators during the sample period, and $\Delta q_{a}(k)$ is the measured change in position of the actuators.

The energy balance, $H$, which is then enforced is implemented as

$$
H=\sum_{k=1}^{n} \Delta H_{I m}(k)+\Delta H_{I s}(k) \geq 0
$$

where $\Delta H_{I m}(k)$ and $\Delta H_{I s}(k)$ are the exchanged amounts of energy at the master and slave side during sample period $\bar{k}$, respectively. (8) is a discrete version of (3) with the interaction points chosen as the actuators of the master and slave device. Enforcing (8) guarantees (4).

\section{COMPlimentarity}

The aim of each of the two approaches discussed in the previous section is complimentary with respect to the aim of the other. The TDPC algorithm enforces passivity of the bilateral controller under all possible operating conditions. In the design phase the transparency of the system is optimized for a set of operating conditions given the boundary condition of passive behavior. The complimentary effect that we wish to establish is to have minimal interference of the TDPC algorithm as long as the operating conditions of the system are within the considered set in the FD design phase. However, when the operating conditions are outside that considered set, the TDPC algorithm should enforce passivity and therefore

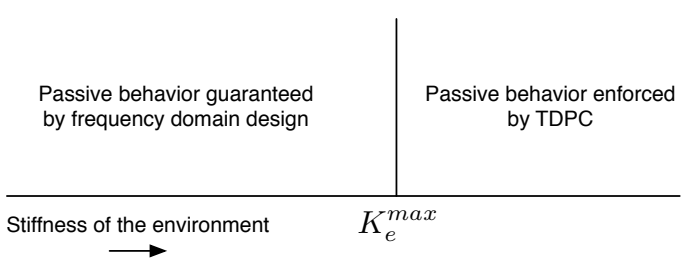

Fig. 1. Desired combination of Bounded Environment Passivity and Time Domain Passivity Control

guarantee stability although the bilateral controller itself is potentially unstable according to the FD-analysis.

This allows optimization of the bilateral controller for a specific set of desired operating conditions. For example in robotic surgery interaction can occur with both soft and hard materials, e.g. tissue and bone, respectively. When the TDPC algorithm is not implemented these hard contacts need to be taken into account in the design phase in the FD. This can impose severe restrictions on the allowable parameter settings and limit the transparency of the system. If a properly matched TDPC algorithm is applied, the considered set of operating conditions in the design phase can be limited to the interaction with soft materials. The stability during hard contacts is then guaranteed by the TDPC algorithm.

In reverse, the application of a TDPC algorithm will by its nature guarantee stable behavior. It does not however convey information about the set of operating conditions that will trigger the TDPC algorithm. To estimate the set of environments that can be accurately displayed by the system without interference, a FD-analysis can be performed.

An example of this desired complimentarity of the two approaches is sketched in Fig. 1. Up to stiffness $K_{e}^{\max }$ passivity of the telemanipulation system is guaranteed by the design of the controller in the FD. For contact stiffnesses higher than $K_{e}^{\max }$ the telemanipulation system as designed in the FD is potentially unstable, but passivity of the system is enforced by the non-linear TDPC algorithm.

\section{Time Domain Passivity Control Extension}

The complimentary effect described in the previous section is not possible with the standard TDPC algorithm. An immediate difference between the approaches of Sections III-A and III-B is that in the FD the device dynamics are incorporated whereas these are neglected in the standard TDPC algorithm. This means that the boundaries of the system between which passivity is designed in the FD differ from those between which passivity is enforced in the TD, Fig. 2. (8) is a more restrictive condition than (4) which means that (8) can indicate that the system is supposedly non-passive, although the system as a whole is still passive.

The TDPC algorithm needs to be adapted to reduce its conservatism. The energy balance which is monitored by the TDPC algorithm could be adapted to correspond with (4) as

$$
\sum_{k=1}^{n} \Delta H_{I h}(k)+\Delta H_{I e}(k) \geq 0
$$

where $\Delta H_{I h}$ and $\Delta H_{I e}$ are the energies exchanged between the user and the master device and between the slave device and the environment, respectively. However, this energy 


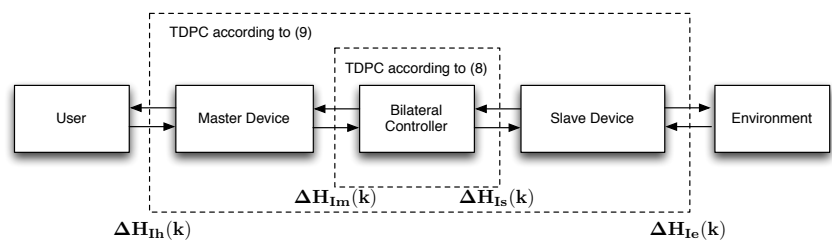

Fig. 2. Schematic overview of energy exchange: Standard TDPC algorithms neglect the device dynamics, whereas these are taken into account in the design phase in the frequency domain.

balance when implemented in a TDPC-algorithm will not produce the desired result, which is guaranteed stability of the interaction with the system. An important difference is that in the FD design phase the passivity of the system is checked when interacting with each considered environment independently. In the TD on the other hand the passivity of the system is monitored online and thus for the interaction with a number of environments and users (e.g. different grasps and/or motions) in series. The history of the interaction can influence the performance of the TDPC algorithm.

The passivity condition of (9) is an inequality, which means that a net amount of energy can be stored in the system when interacting with certain environments. This net injected energy leads to a build-up of energy in (9). When the system starts to interact with an environment for which the FD-analysis showed that the system is non-passive, and thus potentially unstable, this might not be detectable by the TDPC algorithm due to e.g. the prior interaction with other environments. The TDPC algorithm will not be activated until the generated "virtual" energy, due to the nonpassivity of the system, has fully compensated the build-up of energy in (9). Therefore, the interaction with the system could temporarily be unstable. The duration of this temporary potential instability depends on the size of the build up of energy and can be quite significant. An example will be treated in Section VI.

Fig. 3 shows the value for three types of energy balances for a sinusoidal motion with the slave device moving in free space. Fig. 3 shows that the standard TDPC algorithm, based on (8), will be activated even with the slave device moving in free space. However, for a TDPC algorithm based on (9) indeed a build up of energy will occur, which is undesirable with respect to the guaranteed stability of the system. In the following subsections, three extensions to the standard TDPC algorithm will be discussed that will enable the TDPC algorithm to immediately detect non-passive behavior of the system while minimizing its influence when the system itself is passive according to the FD-analysis. The value of this adapted energy balance is also depicted in Fig. 3.

A first extension of the TDPC algorithm was proposed by Franken et al. [15] which incorporate the device friction of the slave device to improve the performance of a TDPC algorithm when applied to telemanipulation systems with significant internal friction at the slave side.

\section{A. Extending the Energy Balance}

The energy balance monitored by the TDPC algorithm can be extended based on the LTI model of the hardware of the telemanipulation system that is used in the FD design phase.

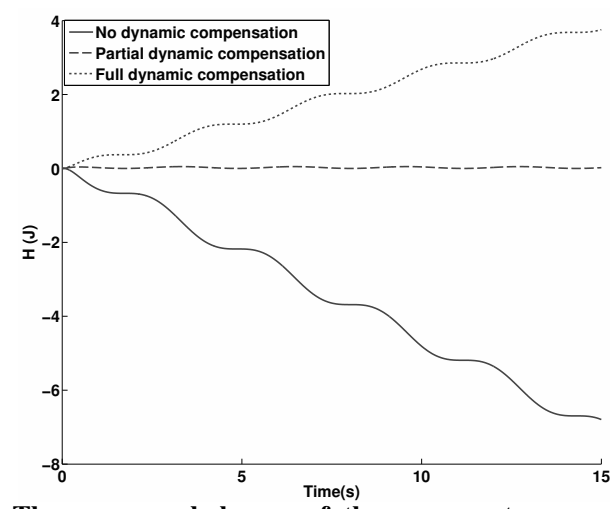

Fig. 3. Three energy balances of the same system moving in free space: without dynamic compensation (8), with full compensation of the device dynamics (9), and the energy exchange at the motors with partial compensation of the device dynamics.

An energy function of each element, e.g. masses, springs, and dampers, can be formulated based on their LTI model. The amount of energy absorbed/dissipated by that element is then a function of the measured displacement. By extending the energy balance of (8) with these additional energy functions the boundaries of the system between which passivity is enforced can be shifted.

In the system given in Section II the master and slave device are both considered to be perfectly rigid arms with a certain mass $M$ and internal friction $B$. This means that the energy exchange of (7) is seperated from the energy exchange between the master and slave device and the slave device and environment as

$$
\begin{array}{r}
\Delta H_{I m}(k)=\Delta H_{I h}(k)-\Delta H_{K m}(k)-\Delta H_{R m}(k) \\
\Delta H_{I s}(k)=\Delta H_{I e}(k)-\Delta H_{K s}(k)-\Delta H_{R s}(k)
\end{array}
$$

where $\Delta H_{K m}(k)$ and $\Delta H_{K s}(k)$ indicate the change in kinetic co-energy of the master and slave device, respectively. $\Delta H_{R m}(k)$ and $\Delta H_{R s}(k)$ indicate the dissipated energy due to viscous friction in the master and slave device, respectively. These energy functions can be derived from the LTI model. A change in kinetic co-energy, $\Delta H_{K}(k)$, can be computed as:

$$
\begin{aligned}
H_{K}(k) & =\frac{1}{2} M \dot{q}(k)^{2} \\
\Delta H_{K}(k) & =H_{K}(k)-H_{K}(k-1)
\end{aligned}
$$

where $H_{K}(k)$ is the kinetic co-energy of the system at sample instant $k$. With the assumption of constant velocity during the sample period, the energy dissipated by viscous friction, $\Delta H_{R}(k)$, becomes

$$
\Delta H_{R}(k)=\frac{B \Delta q(k)^{2}}{\Delta T}
$$

where $\Delta T$ indicates the duration of the sample period. (12) is a conservative estimate of the physically dissipated energy as can be shown using the Cauchy-Schwarz inequality [15].

It should be noted that although this process improves performance (transparent behavior without interference over a larger set of operating conditions), the robustness against modeling inaccuracies is decreased. Underestimating the physical parameters will prevent "virtual" energy from being 
generated.

\section{B. Energy Build-up}

Fig. 3 shows that when the device dynamics are fully compensated according to the previous section, a build up can occur in the monitored energy balance. This is due to the full compensation of the dissipative elements in the system.

As we are extending the energy balance to incorporate the device dynamics, we can choose to not always compensate for the dissipated energy to prevent a build-up. For the compensation algorithm the circumstances need to be identified under which the dissipated energy can be safely compensated. Two methods have been considered, of which one is susceptible to build up under a specific circumstance:

1) Always compensate $\Delta H_{R s}(k)$ and only include $\Delta H_{R m}(k)$ when $H(k)<0$.

2) Compensate $\Delta H_{R s}(k)$ and $\Delta H_{R m}(k)$ only when $H(k)<0$.

where $H(k)$ is the value of the energy balance, and $\Delta H_{R m}(k)$, and $\Delta H_{R s}(k)$ are the dissipated energies in the master and slave device, respectively. The first approach is less conservative as more of the dissipated energy due to physical friction is reclaimed in the energy balance. The first approach works fine for passive environments, but can lead to a build up of energy when motions can be initiated from the environment. The second strategy never leads to a build up, but will cause the TDPC algorithm from being activated more frequently due to the higher amount of neglected energy. Depending on the assumptions made about the environment one of these strategies should be selected.

\section{Energy Scaling}

The telemanipulation system is used to interact with the remote environment. It can be beneficial to not display the forces/velocities in a one-to-one ratio at the master and slave side. Motion scaling can be used to enable high precision micro manipulation capabilities in the remote environment. Motion and force scaling can also be used to design passive behavior of the system, e.g. the use of $\mu \lambda$ in (6) and (5).

When motion and/or force scaling is included in the design of the bilateral controller, the ideal behavior of (1) can be expressed as

$$
\begin{aligned}
\dot{q}_{s}(t) & =\mu \dot{q}_{m}(t) \\
F_{h}(t) & =-\lambda F_{e}(t)
\end{aligned}
$$

This scaling of the power port variables will also effect the energy balance of the system. Taking the scaling values into account the energy balance of the ideal behavior (13) of the two-port system becomes

$$
\int_{t=0}^{t_{1}}-\lambda F_{e}(t) \dot{q}_{m}(t)+\mu F_{e}(t) \dot{q}_{m}(t) d t \geq 0
$$

This shows that unless $\mu=\lambda$ an energy mismatch between the two interaction ports arises. This can either lead to a build up effect as discussed earlier, or cause the modulated damper of the TDPC algorithm to be activated continuously
TABLE I

PARAMETER VALUES OF PHYSICAL SETUP

\begin{tabular}{|l|l||l|l|}
\hline Parameter & Value & Parameter & Value \\
\hline$M_{m}$ & $0.64 \mathrm{~kg}$ & $M_{s}$ & $0.61 \mathrm{~kg}$ \\
\hline$B_{m}$ & $3.4 \mathrm{Ns} / \mathrm{m}$ & $B_{s}$ & $11 \mathrm{Ns} / \mathrm{m}$ \\
\hline$K_{p}$ & $4000 \mathrm{~N} / \mathrm{m}$ & $K_{v}$ & $80 \mathrm{Ns} / \mathrm{m}$ \\
\hline
\end{tabular}

even though the system would be demonstrating the ideal energy behavior.

The solution to this problem is to not enforce two-port passive behavior on the energy balance of the system given by (14). The system does not display two-port passive behavior when scaling is present, but the behavior is passive with respect to a storage function and therefore still mimics a dissipative system, which are thoroughly treated by Willems [16]. That storage function is a transformed energy balance that takes the scaling into account (15). Passivity is being enforced by a modulated damper at the master side, so the energy exchange is normalized with respect to the master side

$$
\int_{t=0}^{t_{1}} F_{h}(t) \dot{q}_{m}(t)+\frac{\lambda}{\mu} F_{e}(t) \dot{q}_{s}(t) d t \geq 0
$$

Naturally this energy balance needs to be adjusted for the build up effect as described earlier. Secchi et al. [17] have similarly applied scaling to haptic interfaces and in a scattering-based telemanipulation algorithm.

\section{EXAMPLE}

In the previous section we have discussed three required extensions to the TDPC algorithm in order to establish complimentarity with the design of the bilateral controller in the FD. To demonstrate the increase of performance of the combined approach several simulations have been performed using the program 20-sim [18].

The system model of Section II is used with the parameters listed in Table I, which are based on the setup described in [6]. The environment consists of a material characterized by a simple spring model with stiffnesses $K_{e}$ located at position $q_{w}=-0.2 m$. The user is modeled as a spring-damper controller with parameters $K_{p}=20 \mathrm{~N} / \mathrm{m}$ and $K_{v}=4 \mathrm{Ns} / \mathrm{m}$ with as set-point a sinusoidal motion with an amplitude of $0.4 \mathrm{~m}$ and frequency of $0.16 \mathrm{~Hz}$.

The modulated damper is implemented as:

$$
\begin{aligned}
F_{\text {pas }}(k) & =-d(k) \dot{q}_{m}(k) \\
d(k) & = \begin{cases}-\alpha H(k) & \text { if } H(k)<0 \\
0 & \text { otherwise }\end{cases}
\end{aligned}
$$

where $F_{\text {pas }}, d(k)$, and $\alpha$ are the additional force applied to the user to restore passivity, the value of the modulated damper, and $\alpha$ is a tuning parameter for the rate at which the additional required energy is extracted from the user, respectively. $\alpha$ is set to 1000 for these simulations to produce a stable contact phase. The sample frequency at which the discrete control loop is computed is $1 \mathrm{kHz}$.

Suppose that based on the task and the particular environment the maximum stiffness that we wish to accurately reflect is $500 \mathrm{~N} / \mathrm{m}$. In that case, using the device parameters 


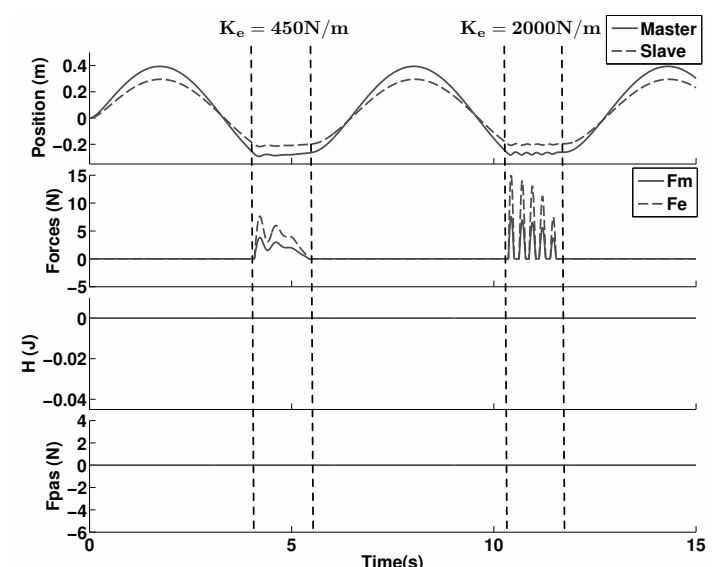

Fig. 4. Position-Force control without TDPC: Stable interaction is not guaranteed with all environments.

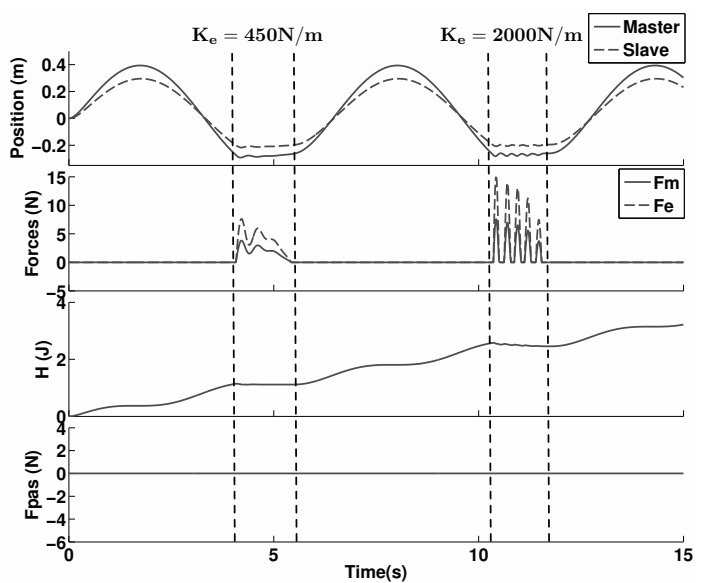

Fig. 6. Position-Force control with incorrect Extended TDPC: Due to an energy build up the algorithm fails to suppress contact instabilities due to active behavior of the bilateral controller.

in Table I and (6), a maximum value holds of $\mu \lambda=0.38$. We select $\mu=0.75$ and $\lambda=0.5$ for good transparency of the telemanipulation system for stiffnesses up to $K_{e}=$ $500 \mathrm{~N} / \mathrm{m}$. However, assume that the hardest contact in the environment has a stiffness of $2000 \mathrm{~N} / \mathrm{m}$. Based on the Bounded Environment Passivity approach a maximum value of $\mu \lambda=0.17$ holds for this worst case stiffness. With the selected settings the telemanipulation system is potentially unstable for certain contacts that can occur during operation.

Several simulations have been performed. In each simulation during the first contact phase the environment stiffness is $K_{e}=450 \mathrm{~N} / \mathrm{m}$ and during the second contact phase the stiffness is $K_{e}=2000 \mathrm{~N} / \mathrm{m}$. Each plot shows the positions of the master and slave device, the force applied to the user $F_{m}$ by the controller and the interaction force at the environment side $F_{e}$. For clarity the level of the monitored energy balance, $H$, and the force exerted by the modulated damper of the TDPC, Fpas, are also plotted separately.

Fig. 4 shows the response of the system when only the designed P-F controller is implemented. The plot demonstrates that for stiffnesses above $K_{e}^{\max }$ stability of the contact between the slave and the environment is not guaranteed by the bilateral controller and the slave device bounces on the surface of the material.

In the second simulation, Fig. 5, the bilateral controller

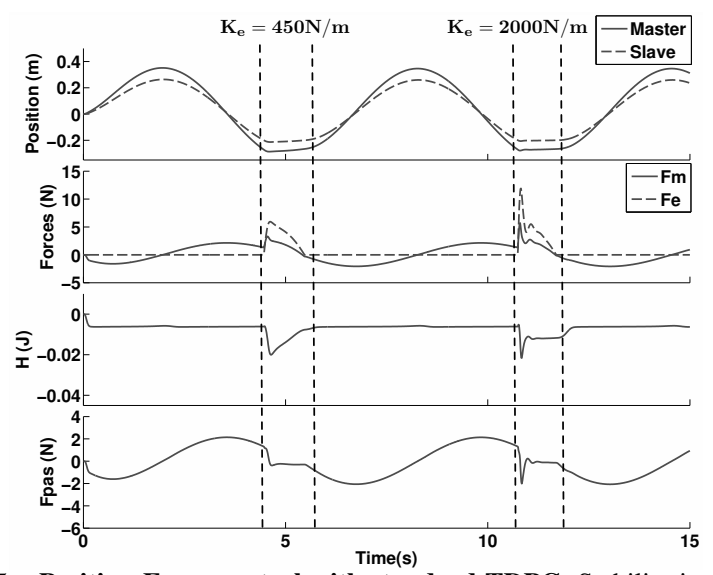

Fig. 5. Position-Force control with standard TDPC: Stability is always guaranteed, but TDPC is also active when stability is already guaranteed by the design of the bilateral controller.

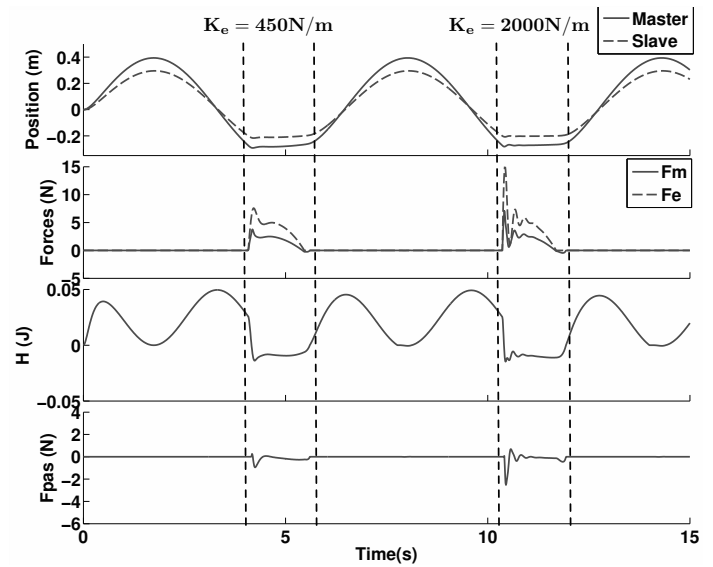

Fig. 7. Position-Force control with correct Extended TDPC: Stability is always guaranteed and the influence of TDPC is minimized when stability is guaranteed by the design of the bilateral controller.

is combined with the standard TDPC algorithm of Section III-B. Fig. 5 shows that the contact stability is improved for $K_{e}>K_{e}^{\max }$, the slave does not bounce on the surface of the material. However, the modulated damper is also highly active during the free space motion and the first contact phase for which stability of the interaction is guaranteed by the FD design phase. This unnecessary additional damping limits the transparency of the telemanipulation system.

In the third simulation the extended TDPC algorithm is used. Fig. 6 shows the system response when the dissipated energy in both the master and slave device is continuously compensated. When the system starts to display non-passive behavior during the second contact phase the modulated damper is not activated due to the positive value of the monitored energy balance. During this period where the bilateral controller generates "virtual" energy the energy balance is decreasing (from $t \approx 10 \mathrm{~s}$ ), but remains positive.

Fig. 7 finally shows the response of the system with the extended TDPC algorithm as proposed in Section V. As no movements can be initiated from the environment, the dissipated energy in the slave device is continuously compensated, see Section V-B. The dissipated energy in the master device is only used when the energy balance is negative. Comparing Fig. 7 with Fig. 4 shows that also with the extended TDPC algorithm the contact stability is 
guaranteed for environments with $K_{e}>K_{e}^{\max }$. Comparing Fig. 7 with Fig. 5 shows that the influence of the extended TDPC algorithm is minimal compared to the standard TDPC algorithm during the free space motion and first contact phase, where $K_{e}<K_{e}^{\max }$. This indicates an increase in the transparency of the telemanipulation system when the extended TDPC algorithm is used.

\section{DISCUSSION}

In the previous section simulation results were presented that show that a better complimentarity between the design of the bilateral controller in the FD and an extended TDPC algorithm can be achieved. However, a perfect complimentarity cannot be established. This is visible in Fig. 6. Even though $K_{e}<K_{e}^{\max }$, the extended TDPC algorithm is active, albeit very lightly.

An important cause for the TDPC algorithm to be activated before the theoretical boundary of $K_{e}^{\max }$, is the neglecting of dissipated energy. This is done to prevent the build up effect. A second cause can be found in the inherent difference between the FD-analysis and TD-implementation. In the FD, a continuous time model of the controller is used, whereas this controller is actually implemented in discrete-time. It is well known that a continuous-time passive model can become non-passive when discretized [19]. However, this effect, given a high enough sample frequency, will only be a minor cause with the used bilateral controller.

Besides the above two causes, which are inherent to the method, a third cause is in this particular example due to the nature of the Bounded Environment Passivity approach. This approach focuses on one-port passive behavior of the interaction between the user and the master device. For light grasps of the user an internal damped resonance mode exists that when excited cause the telemanipulation system to be momentarily two-port non-passive. In that situation the interaction between the user and the master device can remain one-port passive. This difference can be circumvented by applying a FD design method that analyzes passive behavior of both interaction ports, e.g. extending the Bounded Environment Passivity approach with a Bounded Operator approach [6], a similar approach using a different analysis method was proposed by Haddadi et al. [4].

Time-delays in the communication channel have not been considered in this paper. Physical, varying time-delays cannot be taken into account in the FD and even constant timedelays turn any LTI-model into an infinite-dimensional system. The negative influence time-delays have on the stability of bilateral controllers designed for a no-delay situation can be handled effectively by a TDPC algorithm, see e.g. [7], [8]. The transparency properties of the system will be lower compared to the no-delay situation, but stable interaction will still be guaranteed.

\section{CONCLUSIONS}

In this paper it was shown that it is possible to improve the complimentary effect between passivity-based approaches for the design of bilateral controllers in the FD and the enforcing of passive behavior in the TD by means of a TDPC algorithm. Several required extensions to the TDPC algorithm were the addition of the device dynamics to the monitored energy balance, methods for the prevention of an energy build up, and the inclusion of energy scaling. Using these extensions the influence of the TDPC algorithm can be minimized for the set of operating conditions that were considered in the FD design phase. For operating conditions outside this set, and which can potentially destabilize the system, passivity and thus stability is enforced by the TDPC algorithm. Future work will focus on establishing this complimentary effect in physical experiments.

\section{REFERENCES}

[1] D. Lawrence, "Stability and transparency in bilateral teleoperation," IEEE Trans. Robotics and Automation, vol. 9, no. 5, pp. 624-637, 1993.

[2] A. van der Schaft, L2-gain and passivity in nonlinear control. New York: Springer-Verlag, 1999.

[3] N. Hogan, "Controlling impedance at the man/machine interface," Proc. IEEE Int. Conf. Robotics and Automation, pp. 1626-1631, 1989.

[4] A. Haddadi and K. Hashtrudi-Zaad, "Bounded-impedance absolute stability of bilateral teleoperation control systems," IEEE Trans. Haptics, vol. 3, no. 1, pp. $15-27,2010$.

[5] K. Hashtrudi-Zaad and S. E. Salcudean, "Analysis of control architectures for teleoperation systems with impedance/admittance master and slave manipulators," Int. J. Robotics Research, vol. 20, no. 6, pp. $419-445,2001$.

[6] B. Willaert, B. Corteville, D. Reynaerts, H. V. Brussel, and E. B. V. Poorten, "A mechatronic analysis of the classical position-force controller based on bounded environment passivity," Int. J. Robotics Research, pp. 1-18, 2010.

[7] J. Artigas, C. Preusche, G. Hirzinger, G. Borghesan, and C. Melchiorri, "Bilateral energy transfer in delayed teleoperation on the time domain," Proc. IEEE Int. Conf. Robotics and Automation, pp. 671-676, 2008.

[8] M. Franken, S. Stramigioli, R. Reilink, C. Secchi, and A. Macchelli, "Bridging the gap between passivity and transparency," Proc. Robotics: Science and Systems, pp. 281-288, June 2009.

[9] D. Lee and K. Huang, "Passive-set-position-modulation framework for interactive robotic systems," IEEE Trans. Robotics, vol. 26, no. 2, pp. 354-369, 2010.

[10] J.-H. Ryu, D.-S. Kwon, and B. Hannaford, "Stable teleoperation with time-domain passivity control," IEEE Trans. Robotics and Automation, vol. 20, no. 2, pp. 365-373, 2004.

[11] S. Haykin, Active network theory. Addison-Wesley Pub. Co, 1970.

[12] F. B. Llewellyn, "Some fundamental properties of transmission systems," Proc. IRE, pp. 271-283, 1952.

[13] H. Cho and J. Park, "Impedance control with variable damping for bilateral teleoperation under time delay," JSME Int. J. Series C, vol. 48, no. 4, pp. 695-703, 2005.

[14] S. Stramigioli, C. Secchi, A. van der Schaft, and C. Fantuzzi, "Sampled data systems passivity and discrete port-hamiltonian systems," IEEE Trans. Robotics and Automation, vol. 21, no. 4, pp. 574-587, 2005.

[15] M. Franken, S. Misra, and S. Stramigioli, "Friction compensation in energy-based bilateral telemanipulation," Proc. IEEE/RSJ Int. Conf. Intelligent Robots and Systems, pp. 5264-5269, 2010.

[16] J. C. Willems, "Dissipative dynamical systems part i: General theory," Archive for Rational Mechanics and Analysis, vol. 45, pp. 321-351, 1972.

[17] C. Secchi, S. Stramigioli, and C. Fantuzzi, "Power scaling in porthamiltonian based telemanipulation," Proc. IEEE/RSJ Int. Conf. Intelligent Robots and Systems, pp. 1850 - 1855, 2005.

[18] Controllab Products B.V., "20-sim v4.1," http://www.20sim.com/, 2010.

[19] J. Colgate, P. Grafting, M. Stanley, and G. Schenkel, "Implementation of stiff virtual walls in force reflecting interfaces," Proc. iEEE Virtual Reality Annual Int. Symp., pp. 202-208, 1993. 